\title{
Bouts of exercise elicit discordant testosterone: cortisol ratios in runners and non-runners
}

Thiago Paes de Barros De Luccia', José Eduardo Soubhia Natali', Alexandre Moreira², José Guilherme Chaui-Berlinck?' José Eduardo Pereira Wilken Bicudo',3

\begin{abstract}
Objective: The testosterone:cortisol ratio $(\mathrm{T}: \mathrm{C})$ is suggested to be used in order to examine whether physical exercise generates either a "catabolic environment" or an "anabolic environment". The present study aims to evaluate the acute time-course profile of cortisol and testosterone due to an episode of physical exercise. A biphasic profile in the T:C ratio response was hypothesized. Materials and methods: Morning sessions of treadmill running at two different intensities (Heart Rate at $65 \%$ and $80 \%$ of the maximum cardiac reserve) were performed by 6 male non-runners (NR) and 12 trained male runners (subdivided into trained runners T1 and T2). Cortisol and testosterone were measured in saliva. NR andT1 ran for 30 minutes at both intensities, andT2 ran for 46 minutes $( \pm 4.1)$ at $65 \%$ and 42 minutes $( \pm 3.5)$ at $80 \%$. Results: In the $80 \%$ heart rate target, both groups of runners showed the biphasic time-profile, while the non-runners group did not. However, at the $65 \%$ level, none of the groups presented the hypothesized biphasic response. Conclusions: A biphasic timeprofile in the testosterone:cortisol ratio can be seen in short-bout, high intensity exercise (treadmill running) during the morning in men trained for this specific physical activity. Arch Endocrinol Metab. 2018;62(3):325-31
\end{abstract}

Keywords

Testosterone; cortisol; physical endurance

\author{
Departamento de Fisiologia, \\ Instituto de Biociências, \\ Universidade de São Paulo \\ (USP), São Paulo, SP, Brasil \\ ${ }^{2}$ Departamento de Esporte, \\ Escola de Educação Física e \\ Esporte, Universidade de São \\ Paulo (USP), São Paulo, SP, Brasil \\ ${ }^{3}$ School of Biological \\ Sciences, University of \\ Wollongong, NSW, Australia
}

Correspondence to:

Thiago Paes de Barros De Luccia

Departamento de Fisiologia,

Instituto de Biociências,

Universidade de São Paulo

Rua do Matão, 101

05508-090 - São Paulo, SP, Brasil

tpbl78@gmail.com

Received on Oct/31/2017

Accepted on Jan/19/2018

DOI: 10.20945/2359-3997000000042

\section{INTRODUCTION}

$\mathrm{F}$ rom the classic fight-or-flight reaction to subtle dominance relationships in groups, changes are observed in specific hormonal values $(1,2)$. Part of these changes can be understood in terms of their direct metabolic consequences, and both physical and psychological factors seem to play a role as causal factors (1). Although physical exercises are beneficial to health, such activities can generate harmful effects in both men (3) and women (4), a situation that is aggravated by excessive physical exercise $(5,6)$. However, it is not fully known how the beneficial health and fitness-related effects of exercise come to end, subsequently becoming harmful to the human body. The study of hormonal changes related to physical exercise, which have been the focus of much research in the fields of physiology and health, may contribute to this subject (1).

Although there is still no isolated marker capable of diagnosing training problems and/or overtraining (7-9), several indicators have been proposed in recent decades in search of this supposed turning point. One of these markers is the ratio between testosterone, considered an anabolic hormone, and cortisol, considered a catabolic hormone $(10,11)$.

The testosterone:cortisol ratio has been reported as a sign of anabolic status in athletes before competing (12), acute training response $(13,14)$, psychophysiological responses to competition venue (15), training effects (16) and training motivation $(12,17)$. An imbalance between the anabolic and catabolic milien of the metabolism could be associated with certain components of the prescribed exercise (e.g. training volume and intensity) that should be monitored with the intention of improving sports and exercise performance whilst avoiding any deleterious effects from such activity (18).

With regards to acute hormonal responses related to exercise, the testosterone: cortisol ratio $(\mathrm{T}: \mathrm{C})$ has been suggested to be used in order to examine whether physical exercise generates either a "catabolic 
environment" or an "anabolic environment" (19). In a long-term training process, monitoring $\mathrm{T}$ : $\mathrm{C}$ has been advocated in order to verify the hormonal responses to a given training load, which could aid in preventing nonfunctional overreaching or overtraining syndrome (20). In this case, is believed that a catabolic environment might be persistently present (with elevated cortisol and low testosterone concentration) (21), associated with decline in performance, psychological changes, and neuroendocrine disorders.

In general, a low testosterone concentration may be indicative of poor health in men (22), and extreme levels of circulating androgens, whether high or low, can have negative effects on women's health (23). In women, chronic hypercortisolism has been associated with exercise-induced amenorrhea (24). In fact, the analysis of bone mineral density in this group changed the idea of amenorrhea in athletes being a benign event, and linked this phenomenon to premature bone loss associated with a decline in the levels of progesterone and estradiol (25). In a prospective, population-based study of chronic heart disease related to "stress" in men, a low ratio between testosterone and cortisol showed a strong positive association with the components of insulin resistance syndrome (26).

According to some studies, the onset of physical activity stimulates the production of both cortisol and testosterone $(27,28)$. However, cortisol remains elevated in the circulation following the episode of exercise, negatively affecting the synthesis of testicular testosterone (29). These events could generate a biphasic time-profile in the $\mathrm{T}: \mathrm{C}$ ratio, a possible occurrence that has not yet been well studied. These events, which are considered in our hypotheses, are pictorially illustrated in Figure 1 (References used to build the chart (3032 ), in which we show the expected circadian variation in $\mathrm{T}: \mathrm{C}$ and the biphasic response elicited by the physical activity described above.

From this point of view, it is possible to observe that the added effect of acute exercise and the circadian rhythms of testosterone and cortisol obscures our understanding of the biphasic time-profile. Therefore, it is important to analyze the dynamics of these hormones throughout the day. A single isolated measurement might provide a poor picture depending on the sampling phase in relation to the action of cortisol. In fact, this putative biphasic time-profile could play a role in the contradictory data presented in the literature regarding the $\mathrm{T}: \mathrm{C}$ ratio and acute physical activity (33).

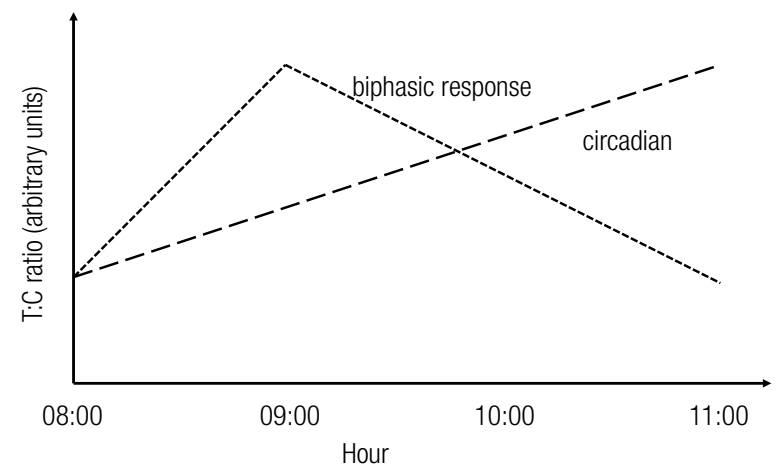

Figure 1. Graphical representation of the $\mathrm{T}: \mathrm{C}$ ratio throughout the morning. The dashed line shows the expected physiological circadian rhythm of T:C. The dotted line represents the hypothesized biphasic response due to a bout of exercise.

\section{Questions in the study}

The present study aims to evaluate the acute timecourse profile of cortisol and testosterone due to an episode of physical exercise, and to answer the following questions:

1) Is there a biphasic profile in the response of the $\mathrm{T}: \mathrm{C}$ ratio?

2) Is there a relationship between this profile and the intensity of acute physical exercise?

3) Is there a relationship between this profile and the duration of acute physical exercise?

4) Is there a difference in this profile between runners and non-runners?

\section{MATERIALS AND METHODS}

\section{Subjects}

Volunteers were selected and divided into two groups in accordance to their previous habitual physical exercise profile (the trained runner group $(\mathrm{T}, \mathrm{n}=$ 12) and non-runner group $(\mathrm{NR}, \mathrm{n}=6))$. The trained volunteers ran an average of $42( \pm 19.2) \mathrm{km} /$ week. They were randomly assigned to two subgroups, $\mathrm{Tl}$ and T2, described below. The non-runner volunteers had varied physical activities but they were not runners. Only male volunteers were selected for the study (mean age $=36$ years $( \pm 8.32)$; mean body mass $=68.8 \mathrm{~kg}( \pm 8.32)$. All participants had a clinical follow-up and signed an informed consent form (the study was approved by the Ethics Committee of the Bioscience Institute - University of Sao Paulo, CAAE 12937713.0.0000.5464). 


\section{Preliminary data collection}

Volunteers visited the laboratory for the first time in order to be physically evaluated and receive instructions about the experimental procedures. Subsequently, each volunteer performed a running session on the treadmill to establish the individual speed corresponding to the target heart rates (see below).

\section{Exercise intensity zones - target heart rates - intensity versus demand}

The exercise intensity was determined by the heart rate reserve method (34):

$$
\mathrm{R}_{\mathrm{p}}=\mathrm{R}_{\text {rest }}+\mathrm{p} \times\left(\mathrm{R}_{\text {max }}-\mathrm{R}_{\text {rest }}\right)(\mathrm{l})
$$

Where $\mathrm{R}_{\text {rest }}$ is the resting heart rate, $\mathrm{R}_{\max }$ is the inferred maximum heart rate of the subject $(220$ - age in years), $\mathrm{p}$ is the desired percentage of the maximum intensity (heart rate reserve) and $\mathrm{R}_{\mathrm{p}}$ is the computed target heart rate.

The protocols used were differentiated by the exercise intensity, the training level of the subjects, and a controlling variable (time or heartbeat) that limits the duration of the exercise. The protocols for each subset are summarized in Table 1.

Table 1. Schematics of the experiments

\begin{tabular}{ccccc}
\hline & Fitness & Non-runners & Trained & Trained \\
\cline { 2 - 5 } & Protocol & Fixed time & Fixed time & $\begin{array}{c}\text { Fixed } \\
\text { heartbeats }\end{array}$ \\
\hline Intensity & $65 \%$ & $\mathrm{NR} \mathrm{R}_{65}$ & $\mathrm{~T} \mathrm{R}_{65}$ & $\mathrm{~T} 2 \mathrm{R}_{65}$ \\
& $80 \%$ & $\mathrm{NR} \mathrm{R}_{80}$ & $\mathrm{~T} 1 \mathrm{R}_{80}$ & $\mathrm{~T} \mathrm{R}_{80}$ \\
\hline
\end{tabular}

The experimental groups were separated into subsets with regards to training level (trained runners vs. non-runners) and the protocol (fixed time vs. fixed heartbeats). Individuals from these subsets performed the exercise at two intensities (65\% vs. $80 \%$ ).

The two exercise intensities selected were $65 \%$ and $80 \%$ of the heart rate reserve $\left(\mathrm{R}_{65}\right.$ and $\mathrm{R}_{80}$, respectively). During the experimental procedures, treadmill speed was adjusted every $5 \mathrm{~min}$ based on the mean heart rate observed within this $5 \mathrm{~min}$ period in order to keep the heart rate as close as possible to the desired target zone.

Each running session for volunteers belonging to the NR or Tl groups comprised a 30 -min run at the individually predetermined $\mathrm{R}_{65}$ and $\mathrm{R}_{80}$. Therefore, these groups performed the exercise at fixed intensities.

The $\mathrm{T} 2$ group performed the exercise in a fixed final energy demand. To impose such a similar final demand within the T2 group, the total number of heartbeats for a running session was fixed. The selected number of beats to be attained was 6,300 (this number of beats resulted in sessions that lasted longer than the 30-min ones completed by the NR and Tl groups, but not so long as to compromise the similarity in the time of day for hormonal collection). From the preliminary data collection, the individual running time at $\mathrm{R}_{65}$ and $\mathrm{R}_{80}$ were computed for each volunteer as the session time $=$ $6,300 / \mathrm{R}_{\mathrm{p}}$. As such, the mean running time at $\mathrm{R}_{65}$ was 46 minutes $( \pm 4.1)$ min and 42 minutes $( \pm 3.5)$ at $\mathrm{R}_{80}$.

\section{Running sessions}

The experiments were conducted at a controlled room temperature of $21( \pm 0.5)$ degrees Celsius. The average relative air humidity was $66 \%( \pm 10 \%)$. Estimated altitude of the laboratory: 785 meters above sea level.

All running sessions were performed in the morning, beginning between 08:00 and 09:00. There were two running sessions, one day at $65 \%$ of the heart rate reserve $\left(\mathrm{R}_{65}\right)$ and the other at $80 \%$ of the heart rate reserve $\left(R_{80}\right)$. The order of the sessions was randomly assigned to each volunteer.

During the running session, the volunteer's heart rate was continuously monitored by surface ECG. The electrodes were attached to the thorax and abdomen (CM5 - a modified Lead I configuration). The ECG was acquired using a sampling rate of $1000 \mathrm{~Hz}$ and standard filters with a MP30 interface and the Biopac Student Lab Pro software (Biopac Systems Inc., Goleta, CA, USA).

\section{Hormone collection and analysis}

The saliva samples for the hormonal analysis were obtained by direct salivation into plastic tubes, stored in a thermal bag at $10^{\circ}$ Celsius and then in a freezer (at $-20^{\circ}$ Celsius).

Samples were taken at three different occasions, at approximately the same time of day, in order to avoid circadian variations.

Basal hormones. Basal samples were collected at 08:00 and 11:00 on days when the volunteer did not perform any physical exercise. The volunteers were required to eat a small meal with around 40 grams of carbohydrate 1 hour before the first saliva sample (07:00).

Experimental hormonal data. On the experimental days, the saliva was collected prior to physical activity (around 08:00 - PRE; the volunteers were instructed to maintain a similar routine pattern as on the day of basal collection), immediately after the bout of exercise 
(around 08:45 - POST), and later on after the activity (around 11:00 - LATE). The volunteers conducted their normal daily activities between the POST and the LATE samples, but were oriented not to ingest large amounts of food.

Salivary cortisol and testosterone were measured using a specific enzyme-linked immunosorbent assay (ELISA) for each hormone (DiaMetra - Salivary Steroid Hormones, Italy; intra-assay variation $\leq 10 \%$ and interassay variation $\leq 8.3 \%$ for the cortisol kit, and intraassay variation $\leq 8.0 \%$ and inter-assay variation $\leq 13.2 \%$ for the testosterone kit). Testosterone was measured in picograms per milliliter, and cortisol in nanograms per milliliter.

\section{Statistical approaches}

First stage: In order to characterize the primary differences between the groups, a one-way ANOVA or two-way ANOVA with repeated measures was employed, as indicated in the results section. Statistical significance was set at $\mathrm{p} £ 0.05$ in this stage.

Second stage: Heart rate. The question of whether the NR group has similar heart rates as the T1 and T2 groups as a whole was addressed by a t-test using the Holm-Bonferroni method for post-hoc significance. A priori, there were four potential comparisons: NR vs. T1; NR vs. T2, NR vs. (T1 and T2), T1 vs. T2. Therefore, the adjusted significance levels (critical p-values) were $0.013,0.017,0.025,0.050$ for the ordered p-values obtained in the comparisons.

Time-profile of the $\mathrm{T}: \mathrm{C}$ ratio. In this case, for each group, there were three potential comparisons: PRE vs. POST; PRE vs. LATE; POST vs. LATE. In a similar manner to the heart rate comparisons, the differences were addressed by a t-test using the Holm-Bonferroni method for post-hoc significance. The adjusted significance levels were $0.017,0.025,0.050$ for the ordered p-values obtained in the comparisons.

\section{RESULTS}

The first step in the analysis was the characterization of the groups based on their heart rates in each running section (Table 2). A one-way ANOVA comparison of the heart rates showed an F-critical (2.15) of 3.68 and the $\mathrm{F}$ values obtained for the $\mathrm{R}_{65}$ and $\mathrm{R}_{80}$ were 5.53 and 3.72 , respectively (p-values of 0.015 and 0.048 ), leading to the conclusion that the groups are dissimilar in their heart rates.
Comparisons between groups (see statistical approaches section) revealed that $\mathrm{NR}$ is different from the $\mathrm{T}$ group as a whole $\left(\mathrm{p}=0.004\right.$ for $\mathrm{R}_{65}$, and $\mathrm{p}=0.013$ for $\mathrm{R}_{80}$ ), while $\mathrm{T} 1$ and $\mathrm{T} 2$ are not different $\left(\mathrm{p}=0.596\right.$ for $\mathrm{R}_{65}$, and $\mathrm{p}=0.887$ for $\left.\mathrm{R}_{80}\right)$.

Therefore, despite the fact that the individuals in the NR group are not sedentary, their heart rates are higher than the those of individuals in the $\mathrm{Tl}$ and $\mathrm{T} 2$ groups, who had trained for running.

Table 3 contains the raw $\mathrm{T}$ : $\mathrm{C}$ ratio data for all the volunteers in the present study. A two-Way ANOVA with repeated measures detected that the $80 \%$ intensity zone generates a difference among the groups (detected as the groups themselves, $\mathrm{p}=0.00004$, and in the sample time, $\mathrm{p}=0.01$ ), while at the $65 \%$ level these differences are not significant.

To complete the analysis and remain on track with the focus of the present study, i.e. whether there is a biphasic time-profile in the $\mathrm{T}$ : $\mathrm{C}$ ratio elicited by physical activity, the time-profile (i.e. PRE, POST and LATE) within each group was characterized as described in the statistical analysis section:

- Non-runner group: there were no differences over time both at the $65 \%$ and $80 \%$ intensity zones.

- Tl runner group: in the $80 \%$ intensity zone, POST > PRE $(\mathrm{p}=0.0059)$.

- T2 runner group: in the 65\% intensity zone, POST > PRE $(\mathrm{p}=0.0231)$ and LATE $>$ PRE $(\mathrm{p}=0.002 \mathrm{l})$, although POST and LATE were not different. In the $80 \%$ intensity zone, POST $>\operatorname{PRE}(\mathrm{p}=0.0083)$.

Figures $2 \mathrm{~A}$ and $2 \mathrm{~B}$ are graphical representations of these results superimposed on the circadian and biphasic response hypothesis depicted in Figure 1.

\section{DISCUSSION}

A biphasic time-profile in the testosterone:cortisol ratio can be seen in short-bout, high intensity exercise (treadmill running) during the morning in men trained for this specific physical activity. Irrespective of the level of training and intensity, it seems that a session of exercise in the morning causes a disruption in the circadian rhythm of the $\mathrm{T}: \mathrm{C}$ ratio. How long such a disruption remains during the rest of the day deserves further investigations. Moreover, whether the modification in the $\mathrm{T}$ : $\mathrm{C}$ ratio promotes a catabolic milieu or not remains an open question, as well as the use of this ratio to evaluate the performance of athletes in acute physical exercise. 
Table 2. Heart rates

\begin{tabular}{|c|c|c|c|c|c|c|c|}
\hline \multirow{2}{*}{ Group } & \multirow{2}{*}{ Subject } & \multicolumn{3}{|c|}{$\mathbf{R}_{65}$} & \multicolumn{3}{|c|}{$\mathbf{R}_{80}$} \\
\hline & & bpm & $\min$ & thb & bpm & $\min$ & thb \\
\hline \multirow[t]{6}{*}{ NR } & 1 & 150 & 30 & 4500 & 164 & 30 & 4920 \\
\hline & 2 & 159 & 30 & 4770 & 169 & 30 & 5070 \\
\hline & 3 & 145 & 30 & 4350 & 161 & 30 & 4830 \\
\hline & 4 & 154 & 30 & 4620 & 163 & 30 & 4890 \\
\hline & 5 & 140 & 30 & 4200 & 153 & 30 & 4590 \\
\hline & 6 & 155 & 30 & 4650 & 160 & 30 & 4800 \\
\hline \multirow[t]{6}{*}{ T1 } & 1 & 146 & 30 & 4380 & 157 & 30 & 4710 \\
\hline & 2 & 139 & 30 & 4170 & 156 & 30 & 4680 \\
\hline & 3 & 133 & 30 & 3990 & 151 & 30 & 4530 \\
\hline & 4 & 128 & 30 & 3840 & 141 & 30 & 4230 \\
\hline & 5 & 123 & 30 & 3690 & 141 & 30 & 4230 \\
\hline & 6 & 135 & 30 & 4050 & 156 & 30 & 4680 \\
\hline \multirow[t]{6}{*}{ T2 } & 1 & 140 & 45 & 6300 & 161 & 39 & 6300 \\
\hline & 2 & 128 & 49 & 6300 & 140 & 45 & 6300 \\
\hline & 3 & 146 & 43 & 6300 & 157 & 40 & 6300 \\
\hline & 4 & 119 & 53 & 6300 & 131 & 48 & 6300 \\
\hline & 5 & 140 & 45 & 6300 & 150 & 42 & 6300 \\
\hline & 6 & 150 & 42 & 6300 & 158 & 40 & 6300 \\
\hline
\end{tabular}

Heart rates (bpm), running time (min) and total heartbeats (thb) for each individual in each protocol (65\% and $80 \%)$.

Table 3. $\mathrm{T}: \mathrm{C}$ data

\begin{tabular}{|c|c|c|c|c|c|c|c|}
\hline \multirow{2}{*}{ Group } & \multirow{2}{*}{ Subject } & \multicolumn{3}{|c|}{$\mathbf{R}_{65}$} & \multicolumn{3}{|c|}{$\mathbf{R}_{80}$} \\
\hline & & PRE & POST & LATE & PRE & POST & LATE \\
\hline \multirow[t]{6}{*}{ NR } & 1 & 11.40 & 17.67 & 13.41 & 13.58 & 13.17 & 25.38 \\
\hline & 2 & 15.12 & 14.75 & 13.59 & 9.68 & 13.85 & 14.76 \\
\hline & 3 & 17.14 & 12.11 & 18.71 & 13.25 & 8.48 & 22.50 \\
\hline & 4 & 11.72 & 15.60 & 11.88 & 18.94 & 23.63 & 15.16 \\
\hline & 5 & 20.80 & 19.31 & 22.58 & 16.87 & 23.46 & 23.64 \\
\hline & 6 & 9.19 & 12.26 & 15.85 & 21.00 & 21.34 & 14.26 \\
\hline \multirow[t]{6}{*}{$\mathrm{T} 1$} & 1 & 10.47 & 15.68 & 9.14 & 12.92 & 14.69 & 10.00 \\
\hline & 2 & 19.46 & 11.84 & 14.74 & 10.20 & 17.37 & 17.30 \\
\hline & 3 & 14.89 & 18.06 & 13.00 & 13.13 & 16.45 & 13.51 \\
\hline & 4 & 16.96 & 17.77 & 17.23 & 11.26 & 15.67 & 15.81 \\
\hline & 5 & 15.92 & 20.05 & 22.39 & 13.35 & 15.27 & 18.13 \\
\hline & 6 & 12.30 & 14.03 & 15.15 & 13.05 & 16.62 & 15.03 \\
\hline \multirow[t]{6}{*}{ T2 } & 1 & 9.09 & 13.32 & 12.36 & 21.40 & 23.23 & 15.84 \\
\hline & 2 & 10.92 & 11.21 & 14.48 & 15.38 & 26.89 & 21.15 \\
\hline & 3 & 9.31 & 17.20 & 16.60 & 13.37 & 23.43 & 24.07 \\
\hline & 4 & 19.76 & 22.11 & 26.52 & 17.64 & 23.11 & 23.57 \\
\hline & 5 & 9.67 & 13.37 & 18.43 & 18.47 & 22.15 & 23.32 \\
\hline & 6 & 12.81 & 14.85 & 16.47 & 17.88 & 23.77 & 15.69 \\
\hline
\end{tabular}

PRE: prior to running; POST: immediately after running; LATE: around 2.5 hours after the running section. 
A

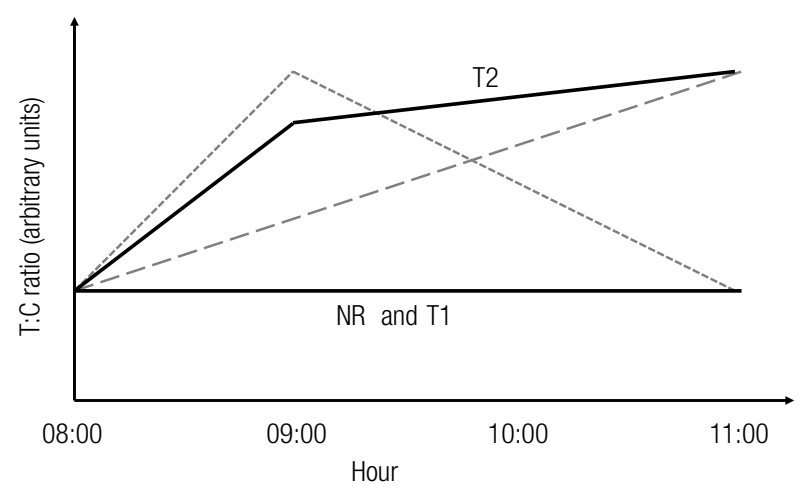

B

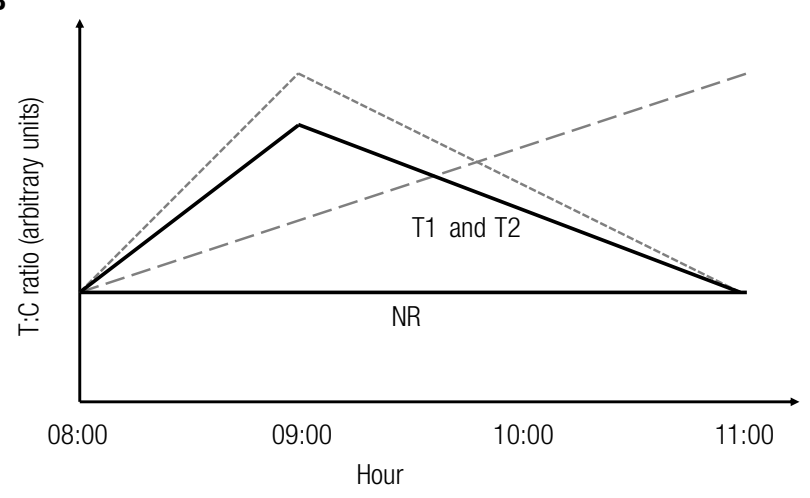

Figure 2. Graphical representation of the T:C results shown in Table 2. (A) Target heart rate of $65 \%\left(R_{65}\right)$. (B) Target heart rate of $80 \%\left(R_{80}\right)$. The curves shown in Figure 1 are in faded gray (circadian and biphasic).

The results of the present study suggest that biphasic behavior in the $\mathrm{T}: \mathrm{C}$ profile seems to depend on the energetic demand of the exercise, as well as the training status of the individuals. Therefore, in the $80 \%$ heart rate target, both groups of runners show the biphasic time-profile, while the non-runner group does not. However, at the $65 \%$ level, none of the groups present the hypothesized biphasic response.

This also answers our questions regarding whether there is an association between the biphasic time-profile and the intensity or duration of the acute physical exercise. The answer is yes, there is such an association between the biphasic time-profile and both intensity and duration, in the sense that biphasic behavior of the $\mathrm{T}: \mathrm{C}$ ratio could only be detected in more elevated levels of demand, i.e. at the $80 \%$ heart rate target.

Our final query was in respect to the putative biphasic time-profile and the individual's training status. The results shown in Figure $2 \mathrm{~B}$ indicate that training specificity plays a role in eliciting a biphasic response.
Here, in a biphasic mode or otherwise, and despite the specific training level, it seems that a bout of acute exercise is able to perturb the circadian rhythm of T:C, reducing the ratio found a few hours after physical activity. An exception for that was the T2 group in the $65 \%$ target, and we have no hypothesis or explanation for this result.

Tremblay and cols. (35) describe a significant increase in cortisol only after 120 minutes of running at $55 \%$ of $\dot{\mathrm{V}}_{\mathrm{O} 2 \max }$ in trained runners (contrasting with running sessions of 40 and 80 minutes). Jacks and cols. (36) evaluated the salivary cortisol responses in 60-minute exercise sessions on a cycle ergometer at three intensities $\left(44.5,62.3\right.$ and $76 \%$ of $\left.\dot{V}_{\text {O2max }}\right)$ in 10 untrained men. The hormone fell in the low and moderate intensities and increased in the high intensity. Despite the intensity, cortisol sampled at 40 minutes during the bouts of exercise showed no significant difference (36). Hoffman (37) describes a dual response of testosterone depending on the duration of the physical activity, with an increase in short bouts (less than $2 \frac{1}{2}$ hours) and a reduction in exercises lasting longer than 3 hours. Therefore, the picture of a "rise and fall" in cortisol and testosterone level is not evident prima facie. Actually, many of these studies do not consider the biphasic time-profile and, as a consequence, additional data acquisition (e.g. at different post-exercise times) could lead to different results.

In a recent meta-analysis (33), the authors concluded that salivary cortisol, and particularly testosterone, are highly influenced by both the study design and sampling time. The present results seem to support this, as described above. In addition, the time (period of the day) of sampling the hormones should be taken into account due to the circadian variation of cortisol and testosterone, both in their circulating levels and in their response curves.

Financial support: this study was supported by a research grant from Fundação de Amparo à Pesquisa do Estado de São Paulo (Fapesp - grant \#2014/14296-1).

Disclosure: no potential conflict of interest relevant to this article was reported.

\section{REFERENCES}

1. Mastorakos G, Pavlatou M, Diamanti-Kandarakis E, Chrousos GP. Exercise and the stress system. Hormones (Athens). 2005;4(2): 73-89. 
2. Czoty PW, Gould RW, Nader MA. Relationship between social rank and cortisol and testosterone concentrations in male cynomolgus monkeys (Macaca fascicularis). J Neuroendocrinol. 2009;21(1):68-76.

3. Gouarné C, Groussard C, Gratas-Delamarche A, Delamarche P, Duclos M. Overnight urinary cortisol and cortisone add new insights into adaptation to training. Med Sci Sports Exerc. 2005;37(7):1157-67.

4. Mountjoy M, Sundgot-Borgen J, Burke L, Carter S, Constantini N, Lebrun $C$, et al. The IOC consensus statement: beyond the Female Athlete Triad--Relative Energy Deficiency in Sport (RED-S). Br J Sports Med. 2014;48(7):491-7.

5. Kim YJ, Lee YH, ParkY, Jee H. Relationship of exercise habits and cardiac events to resting blood pressure in middle-aged amateur marathoners. Gazz Med Ital. 2014;173:273-81.

6. Möhlenkamp S, Lehmann N, Breuckmann F, Bröcker-Preuss M, Nassenstein K, Halle M, et al.; Marathon Study Investigators; Heinz Nixdorf Recall Study Investigators. Running: the risk of coronary events: Prevalence and prognostic relevance of coronary atherosclerosis in marathon runners. Eur Heart $\mathrm{J}$. 2008;29(15):1903-10.

7. Armstrong LE, VanHeest JL. The unknown mechanism of the overtraining syndrome: clues from depression and psychoneuroimmunology. Sports Med. 2002;32(3):185-209.

8. Borresen J, Lambert Ml. Autonomic control of heart rate during and after exercise: measurements and implications for monitoring training status. Sports Med. 2008;38(8):633-46.

9. SmithTB, Hopkins WG, Lowe TE. Are there useful physiological or psychological markers for monitoring overload training in elite rowers? Int J Sports Physiol Perform. 2011;6(4):469-84.

10. Kuhn C. Anabolic steroids. Recent Prog Horm Res. 2002;57:411-34.

11. Christiansen JJ, Djurhuus $\mathrm{CB}$, Gravholt $\mathrm{CH}$, Iversen $\mathrm{P}$, Christiansen JS, Schmitz O, et al. Effects of cortisol on carbohydrate, lipid, and protein metabolism: studies of acute cortisol withdrawal in adrenocortical failure. J Clin Endocrinol Metab. 2007;92(9):3553-9.

12. Cook CJ, Crewther BT, Kilduff LP. Are free testosterone and cortisol concentrations associated with training motivation in elite male athletes? Psychol Sport Exercise. 2013;14(6):882-5.

13. Beaven CM, Gill ND, Cook CJ. Salivary testosterone and cortisol responses in professional rugby players after four resistance exercise protocols. J Strength Cond Res. 2008;22(2):426-32.

14. Beaven CM, Gill ND, Ingram JR, Hopkins WG. Acute salivary hormone responses to complex exercise bouts. J Strength Cond Res. 2011;25(4):1072-8.

15. Arruda AF, Aoki MS, Miloski B, Freitas CG, Moura NR, Moreira A. Playing match venue does not affect resting salivary steroids in elite Futsal players. Physiol Behav. 2016;155:77-82.

16. Gomes RV, Moreira A, Lodo L, Nosaka K, Coutts AJ, Aoki MS. Monitoring training loads, stress, immune-endocrine responses and performance in tennis players. Biol Sport. 2013;30(3):173-80.

17. Cook CJ, Crewther BT. The effects of different pre-game motivational interventions on athlete free hormonal state and subsequent performance in professional rugby union matches. Physiol Behav. 2012;106(5):683-8.

18. Kraemer WJ, Ratamess NA. Hormonal responses and adaptations to resistance exercise and training. Sports Med. 2005;35(4):339-61.

19. Hayes LD, Bickerstaff GF, Baker JS. Interactions of cortisol, testosterone, and resistance training: influence of circadian rhythms. Chronobiol Int. 2010;27(4):675-705.
20. Meeusen R, Duclos M, Foster C, Fry A, Gleeson M, Nieman D, et al.; European College of Sport Science; American College of Sports Medicine. Prevention, diagnosis, and treatment of the overtraining syndrome: joint consensus statement of the European College of Sport Science and the American College of Sports Medicine. Med Sci Sports Exerc. 2013;45(1):186-205.

21. Adlercreutz $H$, Härkönen $M$, Kuoppasalmi $K$, Näveri $H$, Huhtaniemi I, Tikkanen $\mathrm{H}$, et al. Effect of training on plasma anabolic and catabolic steroid hormones and their response during physical exercise. Int J Sports Med. 1986;7 Suppl 1:27-8.

22. Araujo AB, Dixon JM, Suarez EA, Murad MH, Guey LT, Wittert GA. Clinical review: endogenous testosterone and mortality in men: a systematic review and meta-analysis. J Clin Endocrinol Metab. 2011;96(10):3007-19.

23. Davis SR, Wahlin-Jacobsen S. Testosterone in women - the clinical significance. Lancet Diabetes Endocrinol. 2015;3(12):980-92.

24. Olson BR. Exercise-induced amenorrhea. Am Fam Physician. 1989;39(2):213-21.

25. Drinkwater BL, Nilson K, Ott $\mathrm{S}$, Chesnut $\mathrm{CH}$ 3rd. Bone mineral density after resumption of menses in amenorrheic athletes. JAMA. 1986;256(3):380-2.

26. Smith GD, Ben-Shlomo Y, Beswick A, Yarnell J, Lightman S, Elwood P. Cortisol, testosterone, and coronary heart disease: prospective evidence from the Caerphilly study. Circulation. 2005;112(3):332-40.

27. Bloom SR, Johnson RH, Park DM, Rennie MJ, Sulaiman WR. Differences in the metabolic and hormonal response to exercise between racing cyclists and untrained individuals. J Physiol. 1976;258(1):1-18.

28. Wilkerson JE, Horvath SM, Gutin B. Plasma testosterone during treadmill exercise. J Appl Physiol Respir Environ Exerc Physiol. 1980;49(2):249-53.

29. Cumming DC, Quigley ME, Yen SS. Acute suppression of circulating testosterone levels by cortisol in men. J Clin Endocrinol Metab. 1983;57(3):671-3.

30. Bird SP, Tarpenning KM. Influence of circadian time structure on acute hormonal responses to a single bout of heavy-resistance exercise in weight-trained men. Chronobiol Int. 2004;21(1):131-46.

31. Hayes LD, Grace FM, Kilgore JL, Young JD, Baker J. Diurnal variation of cortisol, testosterone, and their ratio in apparently healthy males. Sport SPA. 2012;9:5-13.

32. Hayes LD, Grace FM, Kilgore J, Young JD, Baker J. Salivary hormone response to maximal exercise at two time points during the day. Sport SPA. 2013;10:25-30.

33. Hayes LD, Grace FM, Baker JS, Sculthorpe N. Exercise-induced responses in salivary testosterone, cortisol, and their ratios in men: a meta-analysis. Sports Med. 2015;45(5):713-26.

34. ACSM. ACSM's Guidelines for Exercise Testing and Prescription. J Can Chiropr Assoc. 2013;9:456.

35. Tremblay MS, Copeland JL, Van Helder W. Influence of exercise duration on post-exercise steroid hormone responses in trained males. Eur J Appl Physiol. 2005;94(5-6):505-13.

36. Jacks DE, Sowash J, Anning J, McGloughlin T, Andres F. Effect of exercise at three exercise intensities on salivary cortisol. $J$ Strength Cond Res. 2002;16(2):286-9.

37. Hoffman J. Physiological aspects of Sport training and performance. Florida: Human Kinetics; 2014. 